\title{
Brief introduction of bioinformatics education in China
}

\author{
Q. Dai ${ }^{1,4}$, W. $\operatorname{Liu}^{2,4}$, M. Chen ${ }^{3,4 *}$ \\ ${ }^{1}$ School of Life Science, Zhejiang Sci-Tech University, P.R.China \\ ${ }^{2}$ College of Mathematics, Physics and Electronic Information Engineering, Wenzhou University, P.R.China \\ ${ }^{3}$ College of Life Sciences, Zhejiang University, P.R. China \\ ${ }^{4}$ Bioinformatics Society of Zhejiang Province, P.R. China \\ *e-mail:mchen@zju.edu.cn
}

Key words: education, bioinformatics, international cooperation

As an interdisciplinary field of science and technology, bioinformatics has become an important part of many areas of biology. Bioinformatics in China has grown significantly in the past decade despite a delayed and patchy start at the end of the 1980s by a few pioneer scientists from other disciplines, most noticeably physics and mathematics, where China's traditional strength has been. In the late 1990s and early 2000s, rapid expansion of this field was fueled by the Internet boom and genomics boom worldwide.

In China, more and more researchers are converted into bioinformatics from other disciplines. Meanwhile, with the rapid development of economy and attraction of excellency policy, more and more scientists return China after their formal training overseas and join bioinformatics research. There are more than 30 bioinformatics related societies/organizations are set up to promote the bioinformatics development. More and more scientists from other countries, especially European countries, are drawn to work in China by the improved research and funding environment. International cooperation research institutes/centers are established, e.g. the Max Planck-Chinese Academy of Science Partners Institute in Computational Biology in Shanghai, which employs a number of European scientists and plays key roles in facilitating international collaborations.

Today, more than 30 universities offer undergraduate majors in bioinformatics, and nearly 20 universities offer bioinformatics graduate programs at the $\mathrm{PhD}$ and Master's levels. The number of bioinformatics students is increasing every year. According to a market estimation, average monthly salary for bioinformatics graduates with bachelor degree is about 8,000 RMB Yuan. The country has educated more than 4,000 students, but this is far from meeting the needs of more than 20 thousand of bioinformatics and related companies in China.

In Zhejiang province, Zhejiang University is offering bioinformatics degree programs at the $\mathrm{PhD}$, Master's, and Bachelor's levels. Many other universities have carried out the bioinformatics course, such as Zhejiang Sci-Tech University, Zhejiang University of Technology, Hangzhou Normal University, Zhejiang Chinese Medical University, Wenzhou University, etc. In addition to formal courses, most of the universities offer bioinformatics training program to bench biologists.

The Bioinformatics Society of Zhejiang Province was established in 2013. It is a registered NGO that meets all the legal and financial requirements given by the government. The goal of the society is to provide a platform for bioinformatics scientists, researchers, young students and publics, to create a network between institutions and companies, and to encourage interdisciplinary communication and in-depth discussion. We welcome members from board fields and support members and other persons interested in obtaining advanced knowledge in the field, present current progress and 
expand the general knowledge and applications of bioinformatics. The society will work with foreign societies, contribute to the further education of its members and organize seminars, conferences and workshops. Our next annual meeting will be held this October in Lishui city, Zhejiang.

We work on cooperation in bioinformatics education with Russian universities. We had joint Sino-Russian grant project RFBR-NSCFC (finished in 2016), applied for new research grants in the frames of BRICS initiative in 2018. Recent review papers and special journal issue publications were organized by the international conferences in Russia [1, 2].

Acknowledgements: The participation of MC at BGRS-2018 multiconference has been supported by Russian Ministry of Science project 28.12487.2018/12.1.

\section{References}

1. Orlov Y.L., Fernandez-Masso J.R., Chen M., Baranova A.V. (2018) Medical genomics at Belyaev Conference-2017. BMC Medical Genomics.11(Suppl 1):11. DOI 10.1186/s12920-018-0324-3.

2. Chen M., Harrison A., Shanahan H., Orlov Y. (2017) Biological Big Bytes: Integrative Analysis of Large Biological Datasets. J Integr Bioinform. 14(3). DOI 10.1515/jib-2017-0052. 